\title{
EL FUTURO DE LA COMUNIDAD IBEROAMERICANA DE NACIONES Y LA POLITICA EXTERIOR DE ESPAÑA
}

\author{
Celestino del Arenal (*)
}

\section{CONSIDERACIONES GENERALES.}

Desde el siglo XVI América se ha transformado para España en un constante punto de referencia, sin el cual no sólo no es posible entender su proyección exterior, sino tampoco, sobre todo en determinados momentos, su vida interior. Los casi cuatro siglos de convivencia común, de mestizaje, unidos a los profundos lazos que perduran después de la Emancipación, han pesado y pesan en el devenir de España.

En última instancia, lo que explica este hecho profundo, más sentido, como es lógico, por los españoles que por los americanos, es la conciencia de la existencia de una "comunidad" espontánea, de facto, no articulada, entre los pueblos de habla hispana de las dos orillas del Atlántico, que genera corrientes de simpatía y de solidaridad, por encima de las barreras de todo tipo que nos separan.

En este sentido, desde el momento mismo en que a raiz del proceso de Emancipación se van rompiendo los lazos políticos entre España y América, la existencia de una serie de realidades comunes a España e Iberoamérica, como la historia, la lengua, la cultura e, incluso, ciertos valores comunes, serán la base sobre la que se irá construyendo la idea de la Comunidad Iberoamericana de Naciones. Idea que, filtrada en cada momento histórico a través del tamiz de las diferentes ideologías, intereses y contextos dominantes, se materializará en diferentes modelos concretos de Comunidad.

En consecuencia, la idea de Comunidad y la realidad que la sustenta se han expresado a lo largo de la historia en base a una serie de conceptos y expresiones diferentes, característicos de cada momento histórico y de la filosofía o ideología subyacente, que tienen una diversa significación y alcance y que en algunos casos son consubstanciales con la formulación de un modelo específico de Comunidad.

Los términos que han traducido o traducen la idea de esa Comunidad, y que se han ido acuñando sobre todo a lo largo del siglo XX, son, así, diversos, respondiendo a connotaciones ideológicas y a proyectos de acción exterior muy diferentes. Este es el caso, sobre todo, de los conceptos y expresiones de "Hispanidad", "Comunidad Hispánica de Naciones" y de "Comunidad Iberoamericana de Naciones", que son las más comúnmente utilizados para referirse a la idea y la realidad de esa Comunidad.

Los dos primeros términos, "Hispanidad" y "Comunidad Hispánica de Naciones", vienen a ser, en su definición dominante y más común, dos formulaciones de un mismo modelo de Comunidad, el modelo que hemos denominado tradicional-conservador, que ha ido evolucionando a partir de los años veinte desde los planteamientos propios de la derecha conservadora y tradicional hasta los planteamientos beligerantes del fascismo español (Celestino del Arenal y Alfonso Najera, La Comunidad Iberoamericana de Naciones. Pasado, presente y futuro de la política iberoamericana de España, Madrid, CEDEAL, 1992).

De esta forma, el concepto de Hispanidad enlaza de lleno con la corriente fascista que en los mismos años treinta se está gestando en España. El régimen franquista, por su parte, convertirá la idea de Hispanidad en uno de los objetivos y valores fundamentales de la ideología del Régimen en sus primeros pasos.

Sin embargo, a partir de 1942 y, sobre todo, desde el final de la Segunda Guerra Mundial, el régimen franquista tratará de presentar una nueva versión de la Hispanidad. Esta evolución se reflejará en la aparición de un nuevo término, la "Comunidad Hispánica de Naciones", desarrollada por el Ministro de Asuntos Exteriores, Alberto Martín Artajo. La nueva terminología quiere ser reflejo, dentro del mismo modelo tradicional-conservador de Comunidad, de una versión más atemperada, menos militante, menos agresiva, en consonancia con el nuevo y hostil contexto internacional en que se ve inmerso el régimen franquista a raiz del triunfo de las potencias aliadas en la Segunda Guerra Mundial. En todo caso, el trasfondo ideológico es el mismo.

El término y el concepto de "Comunidad Iberoamericana de Naciones" tiene, por el contrario un sentido y un alcance diferente, en cuanto que viene a expresar un modelo y un proyecto muy distinto de Comunidad. Este modelo es el que se perfila a partir de 1976 con el restablecimiento de la democracia en España. Sus rasgos definitorios reflejan los valores democráticos que lo inspiran, presentando un sentido renovado, cooperativo, solidario, igualitario, alejado de 
las posiciones de preeminencia y autoritarismo que presentaban los modelos anteriores.

\section{IBEROAMERICA Y LA POLITICA EXTERIOR DE ESPAÑA.}

El fin del régimen franquista y el restablecimiento de la democracia en España, bajo la forma de una Monarquía Constitucional, han traido consigo, como se acaba de apuntar, entre otros cambios decisivos, una renovación de la idea y la realidad de la existencia de una Comunidad Iberoamericana de Naciones.

Desde 1976 son numerosas las referencias que se han hecho a esta idea en las declaraciones y discursos oficiales, tanto a nivel de la Corona como a nivel de los distintos gobiernos democráticos que se han sucedido en el gobierno de la Nación. Unas veces con un afán puramente de constatación de una realidad que nos viene impuesta por la historia, otras con un deseo de consolidar esa realidad y avanzar en la articulación práctica de la idea, el hecho concreto es que la idea de una Comunidad Iberoamericana de Naciones se ha ido perfilando en las declaraciones oficiales como uno de los referentes de la proyección exterior de la España democrática. Al lado de la afirmación de la dimensión europea de España, que culmina en 1986 con el ingreso de España en la Comunidad Europea, y como complementaria de la misma, se ha afirmado reiteradamente la dimensión americana de España, como una de las coordenadas que define a España en el mundo.

En este sentido, desde sus primeros esbozos en los inicios de la transición política española hasta sus más recientes expresiones a raiz de la adhesión de España a la Comunidad Europea, la política iberoamericana de España y con ella el modelo democrático de Comunidad Iberoamericana de Naciones han ido experimentando una progresiva maduración, depurando su sentido y alcance y afirmando su raiz democrática y de cooperación.

Lo decisivo en todo este proceso de maduración es que se ha pasado de concebir la política iberoamericana como política de "sustitución", de "legitimación" o de "presión" a considerar esa misma política como política de "adaptación", es decir, como política que debe adaptarse a las realidades de las que parte, España, y hacia las que se orienta, Iberoamérica. De esta forma, se ha pasado de una política contradictoria e irrealista en cuanto al objetivo mismo de afirmar esa Comunidad, a una política coherente y realista en relación con ese mismo objetivo. En definitiva, se ha pasado de una política que trataba de instrumentalizar las relaciones con Iberoamérica en función exclusiva de determinados intereses de régimen o de gobierno a una política de cooperación y concertación, que, inspirada por los principios de solidaridad, igualdad, democracia y desarro1lo, aspira a ser fundamentalmente una política de los pueblos y para los pueblos, basada en los especiales vínculos que unen a España e Iberoamérica.

En este proceso de clarificación y maduración de la política iberoamericana ha jugado, como es lógico, un papel decisivo la progresiva materialización de un modelo global, coherente y realista de política exterior. España, con su integración, primero, en el Consejo de Europa, y después, sobre todo, en la Comunidad Europea, el 1 de enero de 1986 cerraba uno de los capítulos pendientes más importantes en lo que a su definicion y posicionamiento en el mundo se refiere. Se trataba, al mismo tiempo, de la afirmación de una nueva dimensión de su política exterior y de la superación de su tradicional aislamiento respecto de Europa. De esta forma, la presencia española en el mundo no sólo se normalizaba plenamente, sino que además se intensificaba y ampliaba, por efecto de su participación en la política exterior comunitaria.

Algo parecido ha sucedido con lo relativo a la definición de la política de paz y seguridad de España, que era otro de los capítulos pendientes, en su inserción y posicionamiento en el mundo, una vez incorporada a las instituciones europeas. La definición de la política de paz y seguridad se ha cerrado en diciembre de 1989 con la clarificación de los términos de la incorporación a la Alianza Atlántica, el ingreso en la Unión Europea Occidental y la firma de un nuevo Convenio defensivo con los Estados Unidos, basado en la afirmación de una nueva relación bilateral equilibrada. La definición de esta política de paz y seguridad ha normalizado plenamente la posición internacional de España y ha aclarado, igualmente, el sentido y propósitos de su política exterior $y$, en consecuencia, de las relaciones con Iberoamérica.

A partir de diciembre de 1988 , en que se cierran los últimos capitulos pendientes del modelo de politica exterior española, se abre una nueva etapa para la política exterior de España en la que, si los retos y los obstáculos son importantes, como consecuencia del cambio radical que está experimentando el escenario internacional, las bases de partida son ya lo suficientemente sólidas, realistas y coherentes como para que pueda esperarse una respuesta adecuada a los mismos (Celestino del Arenal, "Democracia y politica exterior: el largo camino hacia el cambio", en J. VidalBeneyto (ed.), España a debate, I. La política, Madrid, Tecnos, 1991, pp. 45-65).

En concreto, en el plano de la política iberoamericana, en principio, ya no caben malentendidos. Normalizadas y definidas plenamente su posición internacional y las bases de su acción exterior, la política iberoamericana de España y, con ella, el proyecto de Comunidad Iberoamericana de Naciones pueden afirmarse sin sospechas o recelos en cuanto a la intención última que anima su realización. Tanto los límites como las intenciones de ese proyecto están, o pueden estar, mucho mejor definidos que en el pasado más inmediato.

Es a partir de ese momento cuando realmente, desde la perspectiva española, se puede llevar adelante la idea de Comunidad Iberoamericana de Naciones, en cuanto iniciativa que, partiendo de unas realidades sociales y culturales comunes innegables, de un contexto internacional de creciente y compleja interdependencia, que exige imperiosamente de la cooperación, y de los principios 
democráticos de igualdad, independencia y solidaridad, puede ir progresivamente cristalizando en formas de cooperación y concertación realistas y flexibles, mutuamente beneficiosas para los Estados y pueblos iberoamericanos y español (Celestino del Arenal, "La Comunidad Iberoamericana de Naciones: entre la utopía y la realidad", Revista de Occidente, $\mathrm{n}^{\circ} 131$ (abri1 1992), pp. 163 - 179)

La Cumbre lberoamericana de Guadalajara (México), en 1991, y la Cumbre Iberoamericana de Madrid, en 1992, que han supuesto los primeros pasos en la cristalización e institucionalización del proyecto de Comunidad Iberoamericana de Naciones, defendido por España a partir de 1976, se insertan, sin lugar a dudas, en ese nuevo contexto en el que se mueve la política exterior de España.

El problema de fondo, más alla delas Cumbres lberoamericanas, es que es necesario dar una respuesta adecuada a las perspectivas que se han abierto y saber superar los importantes obstáculos que todavía existen, pues de otra forma se perdería sin lugar a dudas la mejor ocasión en la moderna historia de España para sentar las bases de una auténtica política iberoamericana y de una sentida Comunidad entre los pueblos iberoamericanos y español.

Hoy, en la España democrática y solidaria que vive activamente inmersa en un mundo planetario, interdependiente, complejo y cambiante, la idea de comunidad entre los pueblos americanos de habla hispana y el pueblo español debe continuar siendo una constante de la política exterior de España. Una constante sin la cual no sólo no es posible explicar determinadas posiciones y gestos de España en la política internacional, sino que tampoco plantearse el futuro de España en el mundo.

Desde esta perspectiva, es claro que España, no sólo por razones de solidaridad y cercanía, sino también por razones aunque sea puramente utilitarias, tiene que continuar afirmando y desarrollando su política iberoamericana. Esta constituye un elemento fundamental para romper con los peligros -pérdida de autonomía política, renuncia a actuar como una potencia media con responsabilidades extrarregionales, provincianismo, renuncia a estar presente en el protagonismo futuro de la cultura hispánica, pérdida de la imagen solidaria con los países en vías de desarrollo- que para la política exterior española se derivan de un exagerado eurocentrismo. En el escenario de los años noventa, marcado por el conflicto Norte-Sur, la credibilidad internacional de un Estado democrático va a estar en su posición frente al subdesarrollo y las relaciones Norte-Sur. España debe aprovechar al máximo la proyección multidimensional de sus relaciones internacionales.

España no puede centrar su acción exterior exclusivamente en el ámbito europeo y en el ámbito mediterráneo y del Norte de Africa, por atractiva y sugestiva que pueda ser la tentación y por importante y decisivo que para los intereses estratégicos, políticos y económicos españoles puedan ser dichos ámbitos. Los intereses políticos, sociales y culturales implicados en las relaciones con lberoamérica constituyen elementos de primera importancia en el mundo que se dibuja para principios del siglo $\mathrm{XXl}$, en el que el castellano sera la tercera lengua, con 420 millones de hispanohablantes, y lo hispánico, en sus diferentes manifestaciones, desde lo cultural a lo demográfico, desempeñará un papel decisivo en las relaciones internacionales y en los propios Estados Unidos.

La apuesta decidida por la proyección iberoamericana en vez de debilitar la presencia europea y mediterránea la refuerza, al proporcionar una importante dimensión extrarregional, a nivel político y cultural, que un país de las características de España no está en condiciones de cumplir en ninguna otra región del mundo, fuera de Europa y el Mediterráneo.

\section{LA REALIDAD DE LA COMUNIDAD IBEROAMERICANA DE NACIONES}

Es evidente, por lo tanto, que para España es un punto de interés de primer orden mantener vigentes y profundizar esos vínculos, no sólo como medio de preservar sus propias señas de identidad, sino tambien como factor de presencia y acción solidaria internacional que refuerza su papel en el mundo. No puede extrañar, así, que España haya sido la principal promotora de la idea de Comunidad Iberoamericana de Naciones. Sin embargo, y esto con frecuencia se tiende a ignorar, el desarrollo de esa Comunidad es algo que interesa igualmente a los propios países iberoamericanos, como forma de afirmar tambien sus señas de identidad y reforzar su presencia en el mundo.

Este interés es aún mayor en estos momentos, si tenemos en cuenta que nos encontramos ante una Comunidad que no sólo existe de hecho, en virtud de la existencia de ese entramado de vínculos, sino tambien de derecho, después de la Cumbre Iberoamericana de Guadalajara. Sin embargo, esa Comunidad ya existente es al mismo tiempo un proyecto inacabado y difícil, que se está haciendo día a día, con todos los problemas e intereses, no siempre coincidentes, que suscita todo proyecto ambicioso.

En última instancia, desde la perspectiva española e iberoamericana, ha sido el argumento democrático el que explica el cambio, el renacimiento de la idea y el proyecto de la Comunidad Iberoamericana de Naciones a finales del siglo XX y el que dota de un nuevo sentido al año 1992.

Sin embargo, hay que ser conscientes, que a medio y largo plazo el argumento democrático no es suficiente para impulsar la política iberoamericana de España y edificar ese proyecto de Comunidad, pues no hay democracia política que merezca tal nombre sin desarrollo, sin justicia social, sin democracia económica que la sustente. Es verdad que la España democrática, consciente de esa realidad, ha construido su política Iberoamericana sobre los principios de democracia y desarrollo. Pero también lo es que en la práctica hasta ahora ha primado el apoyo político sobre el económico, produciéndose un desequilibrio que de persistir puede amenazar en el futuro la profundización de las relaciones con Iberoamérica, tanto más cuanto que en el panorama de los años noventa, como consecuencia del cam- 
bio que se está produciendo en el escenario internacional, serán los problemas derivados de las relaciones Norte-Sur los que dominarán el panorama mundial.

Lo anterior nos plantea, por lo tanto, la necesidad por parte del Gobierno de terminar de definir realista y coherentemente el modelo de las relaciones de España con los países en vías de desarrollo y, especialmente, por lo que hace a nuestro objeto de análisis, el modelo de política iberoamericana de España. En última instancia, el futuro de la Comunidad Iberoamericana de Naciones depende de ello. De lo contrario, los buenos propósitos, los cambios habidos en la percepción de las relaciones entre España e Iberoamérica y en la propia política iberoamericana de España y el cambio en la filosofía inspiradora del proyecto de Comunidad no habrán servido de nada.

En todo caso, desde la perspectiva española, la Comunidad Iberoamericana de Naciones, más allá de la importancia que tiene su institucionalización a partir de las Cumbres Iberoamericanas de Guadalajara y Madrid, debe entenderse como un proyecto inspirador de una acción exterior hacia Iberoamérica, que dota a la misma de un alcance y una profundidad que no tienen otras proyecciones exteriores de España.

\section{LA COMUNIDAD IBEROAMERICANA DE NACIONES HACIA EL FUTURO}

Como acabamos de señalar, para que el proyecto de Comunidad Iberoamericana de Naciones pueda llegar a ser en el futuro una realidad, es indispensable que España asuma con todas sus consecuencias el hecho de que la proyección iberoamericana de su política exterior es una dimensión esencial de la misma. Esto supone plantear a fondo la política iberoamericana de España y, en concreto, dar respuesta a una serie cuestiones todavía abiertas.

\subsection{El modelo de política iberoamericana.}

En primer lugar, es absolutamente necesario que se proceda a elaborar, dentro del modelo general de la política exterior española y al igual que se ha hecho con las relaciones con Europa, un modelo definido, coherente y realista de política iberoamericana, con el establecimiento de objetivos a corto, medio y largo plazo y de los medios necesarios para su consecución, que, con el horizonte de principios de siglo y con el punto de impulso de 1992, permita plantear adecuadamente la acción iberoamericana de España.

Nos estamos refiriendo, en consecuencia, a la necesidad de renovar el planteamiento de una política de Estado, a la que se dediquen todos los medios personales y materiales necesarios para su éxito.

Es necesario, en esta misma línea, como se viene haciendo con los Tratados Generales de Cooperación y Amistad que España ha firmado con varios Estados iberoamericanos, continuar avanzando en la puesta en práctica de un enfoque integral a las relaciones con Iberoamérica, de forma que las relaciones político-diplomáticas, económico-comerciales, culturales, cientifico-técnicas y formativas, se ordenen coherentemente de acuerdo con los objetivos planteados.

\subsection{Las bases de partida.}

Este modelo de política iberoamericana debe partir de dos premisas que nos parecen indiscutibles. Una, que la política iberoamericana constituye para España una dimensión esencial de su proyección internacional que es necesario impulsar e intensificar, en paralelo con las dimensiones igualmente esenciales que son Europa y el Mediterráneo / Norte de Africa. Otra, que el contenido del proyecto de Comunidad Iberoamericana de Naciones, que constituye parte de esa politica, debe plantearse especialmente y en primer lugar en términos culturales, pero sin descartar en ningún momento los políticos, pues éstos necesariamente deberán estar presentes desde el primer momento, como forma de sentar las bases mismas del proyecto.

Para todo ello es indispensable, en cualquier caso, la adopcion urgente de una serie de medidas de carácter social y estructural de base, que posibiliten realmente la elaboración, puesta en marcha, desarrollo y constante adecuación de ese modelo de política iberoamericana a las circunstancias cambiantes del entorno mundial e iberoamericano.

En primer lugar, es necesario que, sobre la base de una opinión pública española favorable por principio a la intensificación de las relaciones con Iberoamérica y a la propia política iberoamericana, se plantee una acción de concienciación de la misma a nivel de medios de comunicación sobre lo que supone Iberoámerica y el proyecto de Comunidad Iberoamericana de Naciones, que permita reforzar sobre bases sólidas el consenso que debe caracterizar la política de Estado hacia Iberoamérica.

En segundo lugar, España necesita impulsar urgentemente las investigaciones y estudios sobre Iberoamérica como forma de romper con la dependencia que en este campo existe respecto de los análisis elaborados en los Estados Unidos y otros paises europeos, que no siempre responden a los intereses y planteamientos españoles e iberoamericanos. La politica iberoamericana de España no puede depender de las percepciones que otros países tienen de América Latina, que pueden distorsionar la realidad iberoamericana. Es urgente, en consecuencia, poner en marcha un Instituto o Centro de Estudios sobre Iberoamérica, con los medios humanos y materiales necesarios para cumplir sus objetivos. La falta de recursos que señalamos es una de las claves, aunque no la única, que sirve para explicar algunas de las carencias y contradicciones de la política iberoamericana de España. Sin la creación de ese capital intelectual y humano, a largo plazo la capacidad de España para desarrollar una política iberoamericana activa y coherente se vera seriamente limitada.

En tercer lugar, se plantea la necesidad urgente de realizar un esfuerzo, dentro de las limitaciones que existen, de potenciación y desarrollo tanto de la Dirección General de Iberoamérica del Ministerio de Asuntos Exteriores, dotán- 
dola del personal necesario para atender adecuadamente a lo que debe considerarse es una prioridad de la política exterior, como del gabinete de Análisis y Previsión de ese mismo Ministerio. Es igualmente necesario continuar con la puesta en marcha y desarrollo de la Agencia Española de Cooperación Internacional (AECI), paralizada en los últimos tiempos, ampliando, y no recortando, su presupuesto de cooperación internacional, asi como con la reestructuración, dentro de la AECI, del Instituto de Cooperacidn Iberoamericana (ICI), de forma que éste pueda cumplir con su función fundamental de cooperación con los países iberoamericanos. Paralelamente a las anteriores medidas habría que potenciar algunas de las Embajadas de España en la región, incrementando el personal diplomático.

En línea con lo anterior, es necesario incrementar los niveles de coordinación en materia de cooperación al desarrollo con lberoamerica, no sólo a nivel público, como se viene haciendo por parte de la Secretaría de Estado para la Cooperación Internacional y para lberoamérica (SECIPl) y de la Comisión Interministerial de Cooperación Internacional, sino tambien a nivel público y privado, como forma de obtener la máxima rentabilidad económica y política y la mejor utilización de los recursos.

Finalmente, en este orden de medidas, es indispensable introducir un planteamiento absolutamente realista en la formulación de ese modelo de política iberoamericana. Realismo, primero, en la identificación de los intereses, objetivos y posibilidades de la acción española y, segundo, en función de lo anterior, en cuanto a los medios humanos y materiales que pueden dedicarse a ese fin. Realismo que, por lo tanto, supone tomar en cuenta la importancia de la proyección iberoamericana de España.

En este sentido, es necesario distinguir claramente la retórica de la realidad, las aspiraciones de las posibilidades, como forma de no cometer errores en la planificación de la acción. Es necesario, por lo tanto, interpretar adecuadamente el escenario en que España tiene que moverse, así como los medios y recursos que se tienen para actuar en el mismo, en función, tanto, de la ubicación geo-estratégica y de las características políticas, económicas, científico-técnicas y culturales de una potencia media como España, como en función de los objetivos que se pretenden lograr. Esta leccion, fundamental para no cometer los errores del pasado, parece que afortunadamente se ha aprendido y puesto en práctica en los últimos años a la hora de plantear la política exterior española.

Eso no supone, sin embargo, que haya que eliminar toda retórica. El realismo mismo exige ser conscientes de que la retórica no siempre es algo superfluo, sino en ocasiones algo muy necesario, por cuanto que, al mismo tiempo que es reflejo de las percepciones, contribuye igualmente a crear percepciones y adhesiones, con la importancia que esto tiene en orden a la realización de todo proyecto. Si es verdad que nada hay peor que abusar de la retórica, tambien lo es que no se puede prescindir absolutamente de la misma, con todo lo que tiene de símbolo identificador, generador de opinión pública y guía de un ideal. En concreto, el proyecto de Comunidad Iberoamericana de Naciones, si bien debe plantearse desde perspectivas totalmente realistas, exige retórica, símbolos que faciliten su identificación y construcción.

\subsection{Los intereses en juego.}

El realismo que comentamos supone igualmente la correcta identificación e interpretación de los intereses y conveniencias, tanto propios como recíprocos, tanto comunes como contrapuestos, con el fin de poder plantear una acción eficaz y operativa en base a la adecuada utilización de los medios necesarios para la articulación y satisfacción de esos intereses. Esto vale tanto para las relaciones con los paises iberoamericanos como para las relaciones con los Estados Unidos y la Comunidad Europea en lo que hace a su presencia y actuación en América Latina.

Identificación de intereses que debe realizarse no sólo en base a los factores dominantes en el pasado y en el presente, sino sobre todo tomando en consideración los que se van a producir en el futuro. De ahí, la importancia que tiene el establecer adecuadamente el escenario, lo que nos sitúa de nuevo en la necesidad de poner efectivamente en marcha el Gabinete de Análisis y Previsión del Ministerio de Asuntos Exteriores, así como un adecuado Centro de Estudio e Investigación sobre Iberoamérica. Hay que planificar la acción teniendo en cuenta el nuevo escenario que se está abriendo, así como las nuevas dimensiones que se van añadiendo a la realidad internacional como consecuencia de los importantes cambios que se están produciendo. Hay que estar preparados para ir modificando el modelo al mismo ritmo que se producen los cambios en la realidad, en las percepciones y en las pautas de comportamiento y acción imperantes a nivel internacional.

Identificación de intereses que pasa sin lugar a dudas por la consideración de los intereses comunes y contrapuestos entre los países iberoamericanos y España, cuya obviedad es evidente, pero que pasa tambien por la consideración del papel de la Comunidad Europea y de los Estados Unidos en América Latina y de sus relaciones con España.

La ampliación de los márgenes de autonomía de la política de España hacia Iberoamérica pasa, ante todo, por la clarificación de las relaciones bilaterales con los Estados Unidos, lo que ya se ha producido a partir del 1 de diciembre de 1988 con la firma del nuevo Convenio Defensivo con ese país y con la concreción de los términos de la incorporación de España a la Alianza Atlántica. Pasa también por la continuación del proceso, ya emprendido, de clarificación, entre España y los Estados Unidos de lo que son sus respectivas políticas respecto de Iberoamérica, tanto en lo que se refiere a lo que son intereses comunes como a lo que son intereses contrapuestos o no coincidentes.

Esto en ningún momento debe interpretarse en el sentido de que España deba desarrollar en Iberoamérica una política conjunta con los Estados Unidos, lo que, dada la desigualdad existente a todos los niveles entre ambos países y la existencia de intereses no siempre coincidentes, no sería realista y coherente y se traduciría en una absoluta depen- 
dencia de la política española respecto de la política de los Estados Unidos en la región.

Nuestra posición debe interpretarse en el sentido de que ambos países, como aliados, deben continuar manteniendo los contactos y los canales de comunicación que permitan, como hasta ahora, clarificar y discutir sus respectivas políticas hacia Iberoamerica, a fin de eliminar malentendidos e identificar los intereses comunes y los no coincidentes.

Los intereses e interpretaciones comunes de carácter general son fácilmente identificables, pues tanto los Estados Unidos como España están interesados en la vinculación de América Latina con el sistema occidental, tanto a nivel político como económico y cultural, en la estabilidad política de esos paises y en el desarrollo económico y social de la región y de los distintos Estados. Es sobre estos intereses comunes que debe continuar insistiendo la política iberoamericana de España.

Los intereses e interpretaciones no coincidentes de carácter general también son fácilmente identificables. Estos se centran principalmente en el plano político, social y cultural y sólo muy secundariamente en el plano económico y científico-técnico, dado el carácter poco importante, desde la perspectiva estadounidense, de los flujos económicos, comerciales, financieros y científico-técnicos entre España e Iberoamérica.

Desde un punto de vista político y social, es evidente que España, como se puso claramente de manifiesto en el caso de la crisis centroaméricana, tiene una interpretación de la problemática latinoamericana y unos intereses en la región que no siempre coincide con los de los Estados Unidos. Frente a la interpretación estadounidense de los conflictos y tensiones en términos exclusivamente de mantenimiento del status quo y de la estabilidad, como consecuencia de una caduca concepción de la seguridad nacional, que lentamente empieza a cambiar, la interpretación española considera que la estabilidad y la seguridad de la región, y por lo tanto la solución de sus conflictos, estan inseparablemente vinculadas con la realización de importantes reformas políticas y sociales que permitan a esos países acceder a la democracia real y al desarrollo y progreso económico y social. Ello se ha traducido hasta fechas recientes en una política distinta a la que han seguido los Estados Unidos en América Latina, que ha chocado con frecuencia con sus planteamientos e intereses a corto plazo.

Al mismo tiempo, la política iberoamericana de España $\mathrm{y}$, en concreto, el proyecto de Comunidad Iberoamericana de Naciones responden a unos objetivos de presencia política y cultural en Iberoamérica, basada en la existencia de importantes vínculos históricos lingüísticos, culturales y humanos, que pueden suscitar recelos y chocar en algún momento con los intereses hegemónicos de los Estados Unidos en la región.

No parece, empero, que estos intereses contrapuestos sean un obstáculo real para la política iberoamericana de España y para el objetivo de la Comunidad Iberoamericana de Naciones. La experiencia reciente, y de nuevo la política española en Centroamérica ha sido la piedra de toque, nos demuestra que es posible, desde la posición de aliado en que se encuentra España y desde la toma de conciencia conjunta de los intereses comunes de ambos países, desarrollar una política exterior activa y con importantes márgenes de autonomía en Iberoamérica.

Además, si se confirma el cambio que caracteriza en estos momentos a la política de los Estados Unidos hacia América Latina, incluso una parte importante de esos intereses contrapuestos o no coincidentes pueden empezar a ser comunes y coincidentes. En concreto, la afirmación de los principios de democracia y desarrollo, que hasta ahora han inspirado la política iberoamericana de España, han sido ya asumidos por los Estados Unidos en su política hacia la región.

La necesidad de proceder a una identificación realista de intereses nos situa igualmente ante el reto de considerar e interpretar el papel de la Comunidad Europea en América Latina y los intereses de la misma y de los Estados miembros, como forma de poder obtener el máximo aprovechamiento de la política comunitaria hacia esa región. Identificación que pasa, al igual que en el caso de los Estados Unidos, por la clarificación de los intereses comunes y contrapuestos.

Parece claro que España,dadas las características de potencia media que tiene y sus limitadas posibilidades y recursos de acción en Iberoamérica, si quiere tener cada vez mayor presencia en lberoamérica, desarrollar una política activa en la región y disminuir los riesgos de una eventual tensión con los Estados Unidos en la región, tiene que apoyarse en la Comunidad Europea, europeizando parte de su política iberoamericana.

Este planteamiento sólo será eficaz si previamente se han evaluado correctamente los intereses españoles y comunitarios implicados. Es evidente que América Latina no es una prioridad de la acción exterior ni para la Comunidad Europea ni en principio para el resto de los Estados miembros, como lo es, o lo debe ser, para España, por lo que incluso los intereses comunes que existen entre España, la Comunidad Europea y los demás Estados miembros respecto de America Latina, se transforman con frecuencia en contrapuestos, como consecuencia precisamente de la diferente prioridad que se les atribuye, por un lado, por la Comunidad Europea y sus miembros y, por otro lado, por España. Lo anterior no impide, sin embargo, que España, al europeizar parte de sus relaciones exteriores, se vea afectada por la política comunitaria hacia América Latina, lo que supone que España no puede permanecer indiferente ante la misma y debe tratar de potenciarla.

Al mismo tiempo, dado que, como hemos visto, España tiene sus propios intereses respecto de Iberoamérica, la consideración anterior ratifica la necesidad, obviamente clara, de que España continue con su propia política hacia Iberoamérica. 
Este ha sido afortunadamente el planteamiento seguido hasta ahora por el Gobierno en la elaboración de la política española respecto de Iberoamérica, tanto en el seno de la Comunidad Europea como en la política propia de España. Por un lado, ha intentado por todos los medios potenciar la dimensión europea de su política iberoamericana, tratando de que la Comunidad vaya dedicando cada vez mayor atención a América Latina y haciendo valer los intereses iberoamericanos en las instituciones comunitarias, pues cuanta más atención y preferencia dedique la Comunidad a América Latina será mejor para los intereses españoles en la región, que en virtud de esa dinámica, cada vez serán más comunes y convergentes con los comunitarios, y para los propios intereses iberoamericanos. Por otro lado, trata de desarrollar y profundizar en su propia política iberoamericana.

Lo anterior no debe entenderse en ningún momento en el sentido de que haya que recortar la política europea de España, sino todo lo contrario. Nuestro argumento es que solo impulsando decididamente la presencia y protagonismo de España en Europa, será posible potenciar la política comunitaria hacia América Latina y la política iberoamericana de España podrá desplegarse de forma consistente y operativa en los paises iberoamericanos. Con ello se impulsará igualmente las posibilidades de plasmación del proyecto de Comunidad Iberoamericana de Naciones.

Planteamiento realista, en fin, en la identificación de los intereses en juego con los países iberoamericanos, que debe huir de cualquier intento de generalización de los mismos, más alla de ciertas formulaciones genéricas de principios, dada la gran diversidad de situaciones y problemas, internos e internacionales, que caracterizan a Iberoamérica. Esto es válido no sólo para la política iberoamericana, sino especialmente para una idea y un proyecto tan dificilmente concretable a nivel operativo y práctico como la Comunidad Iberoamericana de Naciones, en el que si en principio, en su formulación genérica, los intereses se presentan como convergentes, llegado el momento de su articulación práctica pueden resultar disímiles y contradictorios en función de la heterogeneidad que caracteriza las distintas realidades e intereses nacionales de los paises iberoamericanos entre si y con España.

\subsection{Los niveles de relación.}

Todo lo anterior nos situa ante la necesidad de fijar los diferentes niveles de relación que deben tomarse en consideración en el modelo de política iberoamericana, a efectos de que este sea realmente operativo y eficaz. Estos niveles, presentes ya, en mayor o menor medida, en la actual política exterior de España, serían los siguientes:

- Nivel global o regional: La política iberoamericana de España dado que parte de una comunidad existente de facto entre España e Iberoamérica, basada principalmente en la historia, la lengua y la cultura, y que tiene entre sus objetivos últimos avanzar en la materialización práctica del proyecto de Comunidad Iberoamericana de Naciones, tiene que tomar en consideración este nivel global, que abarca a toda
Iberoamérica, formulando unas líneas generales de acción, a corto, medio y largo plazo, que contemplen el conjunto de los países iberoamericanos.

Este nivel incluye sobre todo la proyección autónoma de España en Iberoamérica, tanto en el plano genérico como en el plano de las instituciones regionales de integración, cooperación y concertación, caso de la O.E.A., BID, CEPAL, ALADI y SELA, en algunas de las cuales España participa como observadora o invitada, pero incluye tambien, en función de la condición de miembro que tiene España, la proyección de la Comunidad Europea y de los Doce hacia el conjunto de América Latina.

- Nivel subregional. Este nivel responde a la existencia de mecanismos de integración y concertación subregional -Grupo Andino, Centroamérica, Grupo de Riobasados en homogeneidades y dinámicas de distinta naturaleza, que se materializan en actuaciones subregionales más o menos importantes y significativas, respecto de las cuales es necesario con frecuencia una acción específica continuada, especialmente cuando, como sucede en el caso de España, se es miembro observador o invitado en alguna de las mismas. Este nivel se manifiesta igualmente tanto en el plano propiamente español como en el plano comunitario, dadas la especial atención que la Comunidad Europea viene prestando a las relaciones con las subregiones latinoamericanas, como el Grupo Andino, Centroamérica y el Grupo de Rio.

- Nivel bilateral. Responde a las relaciones bilaterales que España ha venido manteniendo con los distintos países iberoamericanos considerados individualmente. La política iberoamericana, partiendo, como es lógico, para evitar contradicciones y discontinuidades, de las políticas puestas en marcha a los niveles regional y subregionales señalados, en lo que afectan a cada país en cuestión, se desarrolla en este caso a un nivel específico y propio respecto de cada Estado, tomando en consideración sus peculiaridades y problemática, asi como los intereses bilaterales implicados, marcándose, en consecuencia, unos objetivos individualizados.

- Nivel transnacional y subnacional. Se trata de un nivel que cada día cobra mayor importancia a la hora de diseñar e implementar una política, dada la creciente intensidad, profundidad y eficacia que tiene las relaciones que se producen en el mismo. En este punto, Iberoamérica presenta una situación que no admite parangón con ninguna otra región del mundo, dada en general la debilidad de los aparatos estatales y la multiplicación consiguiente de numerosas organizaciones subnacionales y transnacionales que tienden a llenar los vacios dejados por los Estados, asumiendo funciones nuevas o funciones que tradicionalmente son propiamente estatales.

A este nivel, la acción española debe materializarse tambien tanto en el plano autónomo como en el plano comunitario, en razón de la política de cooperación que 
la Comunidad Europea realiza a través de las organizaciones no gubernamentales. En el caso de la política iberoamericana de España, la acción a nivel subnacional y transnacional tiene además una particular importancia debido a las propias bases, culturales, lingüísticas y sociales, mas que políticas y económicas, sobre las que sustenta la propia política iberoamericana y el proyecto de Comunidad Iberoamericana de Naciones. Ello hace que tanto la acción de los actores no gubernamentales españoles, sean subnacionales o transnacionales, como las interacciones que se establezcan con los actores iberoamericanos de la misma naturaleza, adquieran una dimensión y una eficacia que no existe en otras situaciones.

\subsection{Los objetivos.}

Tomando en consideración estos cuatro niveles de relación, el modelo de política iberoamericana de España debe establecer unos objetivos generales claros, inspiradores de la acción. Aquí no hay que innovar nada puesto que dichos objetivos ya han sido formulados por el Gobierno, sino simplemente reiterarlos, eso si, con realismo en términos más ambiciosos y más concretos. Estos objetivos, obvios, por otro lado, que se derivan de las consideraciones realizadas hasta ahora, son los siguientes:

I. Incrementar la interdependencia y los intereses comunes entre España e Iberoamérica, desde una perspectiva de solidaridad, como medio de hacer más denso y efectivo el entramado de lazos y vínculos, históricos, sociales, culturales y liguísticos, que ya unen a España e Iberoamérica.

II. Apoyar cuatro principios claves e inseparables, como son paz, desarrollo, democracia e integración, en cuanto inspiradores de toda la política iberoamericana de España. La política española en Iberoamérica debe estar guiada por la búsqueda de la solución pacífica de los conflictos, es decir, por el logro de la paz, lo que supone ineludiblemente el apoyar decididamente el desarrollo económico y social de esos países, la democracia y la integración, sin todos los cuales la paz difícilmente será una realidad.

III. Reforzar el contenido profundo de la relación con lberoamerica, mediante el incremento de la cooperación al desarrollo, entendida en sentido amplio, en todos los planos, económico, cientifico-técnico, etc., sin descuidar el plano cultural, tan importante para el proyecto de Comunidad Iberoamericana de Naciones. Ello requiere indudablemente la existencia de una decidida voluntad política, capaz tanto de arbitrar los recursos necesarios y de profundizar en la política iberoamericana como de movilizar a los agentes privados en orden a que participen activamente en esa política.

IV lmpulsar desde las instancias gubernamentales la intensificación y ampliación de las relaciones comerciales y financieras entre España e Iberoamérica, de forma que se incremente la inversión española, pública y privada, en Iberoamérica y viceversa.

V Facilitar los canales de comunicación y relación de las ONG españolas con Iberoamérica, pues ello reforzará el entramado de vínculos e intereses a niveles a los que no siempre puede llegar la acción gubernamental.

VI Potenciar y desarrollar las relaciones y los mecanismos de enlace y de cooperación entre la Comunidad Europea y América Latina a todos los niveles.

VII Desarrollar mecanismos flexibles, formales o informales, de concertación y cooperación a nivel político, economico, cultural, etc.

VIII Intensificar las relaciones con los sectores y organizaciones indígenas de Iberoamérica.

IX Continuar avanzando en la práctica en el proceso ya abierto de institucionalización de la Comunidad lberoamericana de Naciones.

Los ocho primeros objetivos generales vienen a representar pasos paralelos y/o sucesivos que convergen en la realización del último, pero que tienen sentido en si mismos.

El tercer objetivo señalado, el incremento y la potenciación de la cooperación al desarrollo, en sus distintas manifestaciones, constituye, sin lugar a dudas, uno de los elementos claves sobre los que debe descansar la política iberoamericana y en consecuencia la realización del proyecto de Comunidad Iberoamericana de Naciones, a efectos de hacerlas creibles. Ese objetivo se expresa, además, bajo distintas formas, en el resto de los objetivos generales señalados. Ya hemos indicado anteriormente, que la credibilidad a nivel internacional de la política exterior de un Estado democrático en los años noventa va a depender de su posición y de su respuesta frente a los problemas del subdesarrollo y de las relaciones Norte-Sur. En el caso español esa responsabilidad tiene como una de sus prioridades a Iberoamerica.

Sin embargo, no se puede caer en el error de considerar que la cooperación al desarrollo es el remedio para todos los problemas. La cooperación al desarrollo con ser fundamental no puede, ni debe, sustituir al necesario impulso que hay que dar a las relaciones políticas, culturales, económicas y financieras entre España e Iberoamérica.

\section{CONCLUSION.}

Todas las consideraciones anteriores descansan en el hecho, para nosotros evidente, que señalábamos al principio, de que la proyección iberoamericana de España es una de las dimensiones claves de su política exterior y, en consecuencia, una de las prioridades de su acción exterior. Sólo asumiendo este hecho, que al mismo tiempo constituye un reto para lo que será la política exterior española después de 1992, el proyecto de Comunidad Iberoamericana de Naciones, que ya ha dado sus primeros pasos, continuará con su proceso de consolidación y desarrollo. 
En este sentido, el problema no es la Cumbre Iberoamericana de Madrid, de julio de 1992, en cuya preparación España ha puesto todo su empeño para que la misma sea un éxito y represente un avance en el proceso de afirmación de la Comunidad Iberoamericana de Naciones, sino el después de 1992.

El gran desafío que tiene planteada la política exterior española a partir de 1992, al margen del proceso de convergencia con los países de la Comunidad Europea y del avance de la Unión Europea, es que Iberoamérica no deje de ser una de las dimensiones prioritarias de su acción exte- rior. Si hasta ahora el compromiso de 1992 ha actuado como un reto en el desarrollo de las relaciones de España con Iberoamérica, se corre el peligro de que, pasada esa mítica fecha, la política exterior española, acuciada por los retos europeos y preocupada por la evolución del Magreb, sitúe la política iberoamericana en un segundo plano, conformándose con mantener, y renunciando a ampliar y profundizar, unas relaciones que desde 1976, han conocido un impulso y una intensificación desconocidos anteriormente que han permitido, junto a otros hechos, que España recupere un prestigio y un protagonismo internacional que no tenía desde hacía mas de dos siglos.

\title{
RESUMEN
}

La proyección iberoamericana de España es una de las dimensiones claves de su política exterior y, en consecuencia, una de las prioridades de su acción exterior. Sólo asumiendo este hecho, que al mismo tiempo constituye un reto para la futura política exterior española después de 1992, el proyecto de Comunidad Iberoamericana de Naciones, continuará con su proceso de consolidación y desarrollo.

\begin{abstract}
The iberoamerican dimension of Spain is one of the key aspects of the Spanish Foreign Policy, and as a consequence, one of the priorities of its foreign action. From this point of view, which constitutes at the same time a challenge for the future Spanish Foreign Policy after 1992, the project of a "Comunidad Iberoamericana de Naciones" will continue with its process of development and consolidation.
\end{abstract}

